УДК 624.13

${ }^{1}$ C. В. Босаков, д-р техн. наук, ${ }^{2}$ О. В. Козунова, канд. техн. наук

\title{
РАСЧЕТ ШАРНИРНО-СОЧЛЕНЕННЫХ ПРЯМОУГОЛЬНЫХ ПЛИТ НА УПРУГОМ ОСНОВАНИИ
}

Рассматривается задача статического расчета шарнирно-сочлененных в отдельных точках прямоугольных плит понтонных переправ на основании Винклера, дорожных и аэродромных плит на упругом полупространстве и комбинированном основании Винклера-Когана на произвольную нагрузку. Смежные плиты соединяются цилиндрическими шарнирами. Расчет выполнен смешанным методом строительной механики с использованием способа Жемочкина и учетом собственного веса каждой плиты.

Ключевые слова: статический расчет, прямоугольная плита, упругое основание, способ Жемочкина.

Введение. Задача статического расчета шарнирно-сочлененных прямоугольных плит на упругом основании в полной мере и универсальной постановке не решена до настоящего времени. Исследованиями по расчету шарнирно-соединенных плит на упругом основании занимались в СССР: Б. Г. Коренев [8], который предложил решение этой задачи с использованием обобщенных функций; Р. В. Серебряный [11] - на основании своего подхода дал решение пространственной задачи об изгибе шарнирно-соединенных прямоугольных плит на упругом полупространстве и составил таблицы для облегчения инженерных расчетов; Г. Я. Попов [9] получил точное решение контактной задачи для шарнирно-сочлененных балочных плит на упругой полуплоскости, но при этом он использовал сложный математический аппарат для получения этого решения; А. Г. Юрьев [12] использовал интегральный метод Л. П. Винокурова для решения этой задачи.

Общий подход для статического расчета шарнирно-соединенных жестких прямоугольных плит на упругом основании, основанный на смешанном методе строительной механики [10] и способе Б. Н. Жемочкина [6], был описан в работе авторов [1]. Этот подход позволяет с единых позиций рассчитывать шарнирно-соединенные плиты или балки любой формы и жесткости на различных моделях упругого основания на произвольную вертикальную нагрузку. Численная реализация предлагаемого подхода выполнена на семи шарнирно-соединенных прямоугольных плитах на основании Винклера и приведена в [1].

Модель упругого основания в виде модели трехслойного основания, или в дальнейшем будем называть ее моделью комбинированного основания Винклера - Когана, была предложена в работе авторов [8] для

(C) С. В. Босаков, О. В. Козунова, 2019 
расчета изолированной дорожной плиты автомобильных дорог и аэродромов на неоднородном (слоистом) основании. Модель такого основания представима в виде основания Винклера (слой щебня), расположенного на двухслойном основании Когана (песок + естественный грунт).

Используя результаты работы Б. И. Когана [7] и С. В. Босакова [3] об определении перемещений двухслойного основания, а также подход Б. Н. Жемочкина [6] о расчете жесткой плиты на двухслойном основании, авторами в [2] было получено выражение для определения перемещений точки поверхности трехслойного основания от действия нагрузки, равномерно распределенной по прямоугольному участку поверхности трехслойного основания.

Комбинированная модель Винклера - Когана приближена к реальным геологическим условиям строительной площадки и может быть использована для расчета шарнирно-сочлененных прямоугольных плит на неоднородных (слоистых) основаниях.

Постановка задачи. Рассмотрим систему из $N_{p}$ шарнирно-сочлененных в двух точках прямоугольных плит размерами $2 a \times 2 b$ на упругом основании (рис. 1) под действием внешней нагрузки. Крайние плиты будем считать шарнирно опертыми с одной стороны на неподвижные опоры.

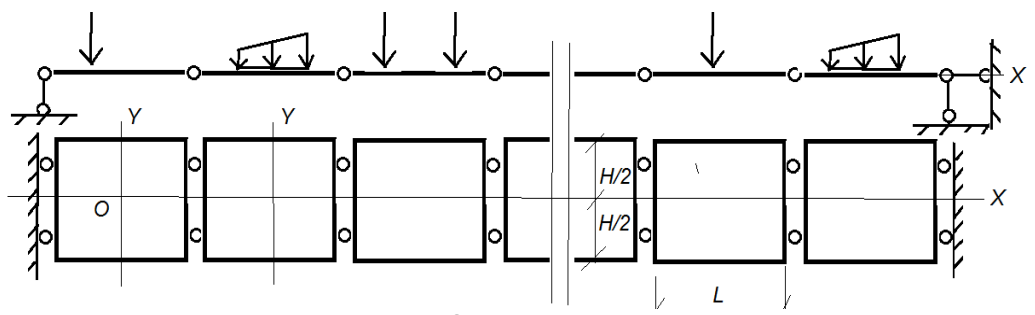

Рис. 1 - Заданная система шарнирно-соединенных прямоугольных плит

Каждую плиту разобьем на одинаковые прямоугольные участки размерами $\Delta x \times \Delta y$ и в центре каждого участка поставим вертикальную связь, через которую осуществляется контакт плиты с упругим основанием. Будем считать, что усилие в связи вызывает равномерную эпюру давлений в пределах участка.

Вводим следующие условия контактного взаимодействия:

- плиты работают совместно с упругим основанием, без отрыва и горизонтального смещения;

- силами трения в контактной зоне плиты с упругим основанием пренебрегаем (отсутствуют касательные напряжения);

- для плиты справедливы гипотезы технической теории изгиба [10].

Полученную статически неопределимую систему решаем смешанным методом строительной механики [10], приняв за неизвестные: усилия во введенных вертикальных связях, линейные и угловые перемещения защемления в центре каждой плиты, поперечные силы в соединительных шарнирах. 
На рис. 2 приводится фрагмент основной системы смешанного метода для крайних плит.
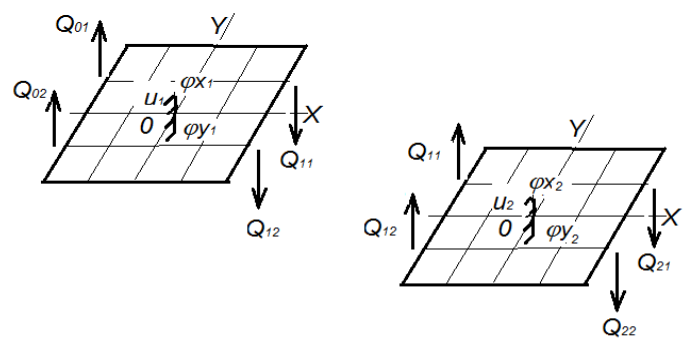

Рис. 2 - Левый фрагмент основной системы

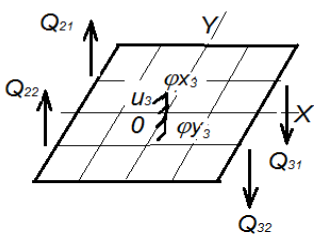

смешанного метода строительной механики

Канонические уравнения смешанного метода для средней плиты с номером $i$ запишем в следующем виде

$$
\left\{\begin{array}{l}
\sum_{k=1}^{2} \delta_{Q, i-1} Q_{i-1}^{k}+\delta_{1,1} X_{1}+\ldots+\delta_{1, m} X_{m}+u_{i}+\varphi x_{i} y_{1}+\varphi y_{i} x_{1}+\sum_{k=1}^{2} \delta_{Q, i} Q_{i}^{k}+\Delta_{1, p}=0 \\
\ldots \\
\sum_{k=1}^{2} \delta_{Q, i-1} Q_{i-1}^{k}+\delta_{m, 1} X_{1}+\ldots+\delta_{m, m} X_{m}+u_{i}+\varphi x_{i} y_{m}+\varphi y_{i} x_{m}+\sum_{k=1}^{2} \delta_{Q, i} Q_{i}^{k}+\Delta_{m, p}=0 \\
\sum_{k=1}^{m} X_{k} y_{k}-\frac{H}{2} Q_{i, 1}+\frac{H}{2} Q_{i, 2}+\frac{H}{2} Q_{i-1,1}-\frac{H}{2} Q_{i-1,2}-M_{x}=0 \\
\sum_{k=1}^{m} X_{k} x_{k}-\frac{L}{2} Q_{i, 1}-\frac{L}{2} Q_{i, 2}-\frac{L}{2} Q_{i-1,1}-\frac{L}{2} Q_{i-1,2}-M_{y}=0 \\
\sum_{k=1}^{m} X_{k}-Q_{i, 1}-Q_{i, 2}+Q_{i-1,1}+Q_{i-1,2}-R=0 ; \\
\sum_{k=1}^{2} \delta_{Q, i-1} Q_{i-1}^{k}+u_{i-1}-\frac{h}{2} \varphi x_{i-1}-\frac{L}{2} \varphi y_{i-1}+u_{i}-\frac{h}{2} \varphi x_{i}+\frac{L}{2} \varphi y_{i}=0 \\
\sum_{k=1}^{2} \delta_{Q, i-1} Q_{i-1}^{k}+u_{i-1}+\frac{h}{2} \varphi x_{i-1}+\frac{L}{2} \varphi y_{i-1}+u_{i}+\frac{h}{2} \varphi x_{i}-\frac{L}{2} \varphi y_{i}+\sum_{k=1}^{2} \delta_{Q, i} Q_{i}^{k}=0
\end{array}\right.
$$

где $m$-число участков Жемочкина на плите; $u_{i}, \varphi x_{i}, \varphi y_{i}-$ неизвестные линейное и угловые перемещения введенного защемления на плите с но- 
мером $i ; R, M_{x}, M_{y}$ - равнодействующая внешних сил и моменты равнодействующей относительно координатных осей на плите с номером $i$; $Q_{i, 1}, Q_{i, 2}$-поперечные силы в разрезанных шарнирах по одну правую сторону плиты с номером $i$; $X_{k}$ - усилие в связи Жемочкина с номеpom $k$.

Коэффрициенты при неизвестных уравнения (1) определяются из следующих соотношений:

а) для основания Винклера, из работы авторов [1],

$$
\begin{aligned}
\delta_{i, k} & =\frac{1}{k \Delta x \Delta y}+\frac{b^{2}}{16 \pi D} w_{i, k}, \quad i=k ; \\
\delta_{i, k} & =\frac{b^{2}}{16 \pi D} w_{i, k}, \quad i \neq k,
\end{aligned}
$$

где $k$ - коэффрициент постели упругого основания, определяется [11] по формуле

$$
k=\frac{E_{0}\left(1-v_{0}\right)}{\left(1+v_{0}\right)\left(1-2 v_{0}\right) h_{0}}
$$

справедливой для решения плоской задачи теории упругости, как показали исследования [12]: при толщине упругого слоя $h_{0} \leq 0,5 b$ (практически точно), $h_{0} \leq 0,75 b$ (с точностью до $5 \%$ ), $h_{0} \leq b$ (с точностью до $8 \%$ ); где $b$ - полуширина плиты, $E_{0}, v_{0}$-упругие параметры основания;

б) для упругого полупространства

$$
\delta_{i, k}=\frac{1-v_{0}^{2}}{\pi E_{0} \Delta x} F_{i, k}+\frac{b^{2}}{16 \pi D} w_{i, k},
$$

где $F_{i, k}$ - безразмерная функция, определяется [6] по формуле

$$
F_{i, k}=2 \frac{\Delta x}{\Delta y}\left[\ln \frac{\Delta y}{\Delta x}+\frac{\Delta y}{\Delta x} \ln \left(\frac{\Delta x}{\Delta y}+\sqrt{\frac{\Delta x^{2}}{\Delta y^{2}}+1}\right)+\ln \left(1+\sqrt{\frac{\Delta x^{2}}{\Delta y^{2}}+1}\right)\right]
$$

в) для комбинированного основания Винклера - Когана.

В работе авторов [2] рассмотрена конструкция грунтового основания в виде поверхностного слоя щебня, расположенного на слое песка, который, в свою очередь, находится на естественном грунтовом полупространстве. Модель такого основания представима в виде основания 
Винклера (слой щебня), расположенного на двухслойном основании Когана (песок + естественный грунт) [7] (рис. 3).

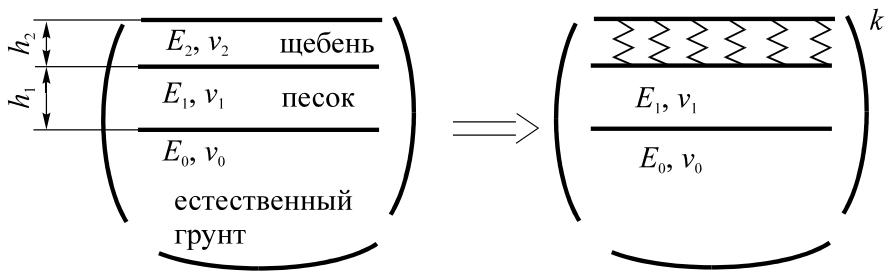

Рис. 3 - Модель комбинированного основания Винклера - Когана

Авторами [2] приведено выражение для определения перемещений точки $M\left(x_{i}, y_{i}\right)$ поверхности трехслойного основания от действия равномерно распределенной по прямоугольному участку размером

$$
\left(x_{k} \pm \Delta x / 2\right) \times\left(y_{k} \pm \Delta y / 2\right)
$$

поверхности трехслойного основания от действия единичной нагрузки в следующем виде

$$
W\left(x_{i}, y_{i}\right)=\frac{P\left(1-v_{1}^{2}\right)}{\pi E_{1} h_{1}} F_{i, k} .
$$

где $F_{i, k}$ - безразмерная функция, которая определяется по фрормулам, полученным авторами [2] с учетом соотношений [6, 7, 2], а именно

$$
\begin{gathered}
F_{i, i}=\frac{1}{k \Delta x \Delta y}+2 \frac{\Delta x}{\Delta y}\left[\ln \frac{\Delta y}{\Delta x}+\frac{\Delta y}{\Delta x} \ln \left(\frac{\Delta x}{\Delta y}+\sqrt{\frac{\Delta x^{2}}{\Delta y^{2}}+1}\right)+\ln \left(1+\sqrt{\frac{\Delta x^{2}}{\Delta y^{2}}+1}\right)\right]+ \\
+\sum_{n=0}^{3} a_{n} \frac{\Gamma(n+1)}{\left(4+R^{2} / h_{1}^{2}\right)^{\frac{h+1}{2}}} P_{n}(z)\left(\frac{2 h_{1}}{\sqrt{R^{2}+4 h_{1}^{2}}}\right) \\
F_{i, k}=\frac{h_{1}}{R}+\sum_{n=0}^{3} a_{n} \frac{\Gamma(n+1)}{\left(4+R^{2} / h_{1}^{2}\right)^{\frac{h+1}{2}}} P_{n}(z)\left(\frac{2 h_{1}}{\sqrt{R^{2}+4 h_{1}^{2}}}\right) \\
R=\sqrt{\left(x_{i}-x_{k}\right)^{2}+\left(y_{i}-y_{k}\right)^{2}} .
\end{gathered}
$$

В приведенных выше формулах: $Г(n+1)$-Гамма-фрункция; $P_{n}(z)-$ полином Лежандра [10]; $k$-коэфрфициент постели верхнего слоя ком- 
бинированного основания: основания Винклера, который определяется [4] по фрормуле, аналогичной фрормуле (3), а именно

$$
k=\frac{E_{2}\left(1-v_{2}\right)}{\left(1+v_{2}\right)\left(1-2 v_{2}\right) h_{2}},
$$

где $E_{2}, v_{2}$ - модуль упругости и коэффрициент Пуассона верхнего слоя комбинированного основания (щебня, рис. 3); при рекомендуемой исследованиями [5] толщине упругого слоя (см. выше).

Коэфффициенты $a_{n}(n=0,1,2,3)$ в формулах (7), (8) определяются из соотношений, которые получены одним из авторов в монографии [2] для двухслойных оснований, а именно:

$$
\begin{aligned}
& a_{0}=\frac{M+L}{M-L-S}-1 ; \\
& a_{1}=2 a_{0}+\frac{2 L(S-2 N)-2 M(S-2 N)-4(2 L M+N S)}{(L-M+S)^{2}} ; \\
& a_{2}=2 a_{1}-2 a_{0}-2 \frac{(S-2 N)\left(M^{2}-L^{2}\right)+\left(8 L M+2 N S+S^{2}\right)(L+M)}{(L-M+S)^{3}} ; \\
& a_{3}=e^{2} \frac{L / e^{4}+M+4 N / e^{2}}{M-L / e^{4}-(4 N+S) / e^{2}}-e^{2}-a_{0}-a_{1}-a_{2} .
\end{aligned}
$$

В формулах (10) используются новые параметры, выраженные соотношениями [7] через упругие характеристики (модуль упругости и коэффициент Пуассона) для каждого упругого слоя рассматриваемого двухслойного основания, а именно:

$$
\begin{aligned}
& M=\left(3+m-4 v_{1}\right)\left(4 m v_{0}-1-3 m\right) ; \\
& L=(1-m)\left(3-3 m-4 v_{1}+4 m v_{0}\right) ; \\
& N=(1-m)\left(1+3 m-4 m v_{0}\right) ; \quad m=E_{1}\left(1+v_{0}\right) / E_{0}\left(1+v_{1}\right) ; \\
& S=2\left[(1-m)(5+3 m)+4 v_{1}\left(m-3+2 v_{1}\right)+4 m v_{0}\left(1+m-2 v_{1}\right)\right] .
\end{aligned}
$$

Прогибы плиты с защемленной нормалью (рис. 4) в основной системе смешанного метода в полярных координатах определяются [2] по формуле

$$
\begin{aligned}
w(r, \theta)= & \frac{P b^{2}}{16 \pi D}\left(\frac{d^{2}-2 d r \cos \theta+r^{2}}{b^{2}} \ln \frac{d^{2}-2 d r \cos \theta+r^{2}}{b^{2}}+\right. \\
& \left.+\frac{2 d r \cos \theta}{b^{2}}\left(\ln \frac{d^{2} r^{2}}{b^{4}}-1\right)-\frac{d^{2}}{b^{2}} \ln \frac{d^{2}}{b^{2}}-\frac{r^{2}}{b^{2}} \ln \frac{r^{2}}{b^{2}}\right),
\end{aligned}
$$


где $d$ - радиальная координата точки приложения сосредоточенной на плите; $D$ - цилиндрическая жесткость плиты.

Первые $(m+3)$ системы $(1)$ подобны стандартным уравнениям способа Жемочкина [6], где дополнительно входят 4 поперечные силы в разрезанных шарнирах. Последние два уравнения выражают условия отсутствия вертикальных взаимных перемещений в местах расположения промежуточных шарниров на границе двух соседних плит. Для первой и последней крайних плит в системе (1) два уравнения выражают условия отсутствия вертикальных перемещений в опорных шарнирах.

Если обозначить число плит через $N_{p}$, то общее число неизвестных усилий в связях Жемочкина, линейных и угловых перемещений введенных защемлений на плитах и поперечных сил в шарнирах для расчета этой системы плит выразится формулой

$$
N p(m+3)+2 N p+2 \text {. }
$$

Структура системы разрешающих уравнений для системы шарнирно-соединенных плит представлена на рис. 5. Блоки по главной диагонали образованы по системе (1), побочные блоки являются нулевыми в случае основания Винклера. В случае упругого полупространства и комбинированного основания Винклера - Когана побочные блоки - ненулевые и характеризуют взаимное влияние плит, см. формулы (5), (7), (8).

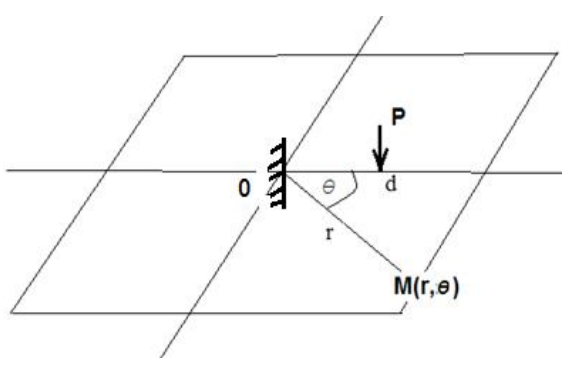

Рис. 4 - Плита с защемленной нормалью в полярных координатах

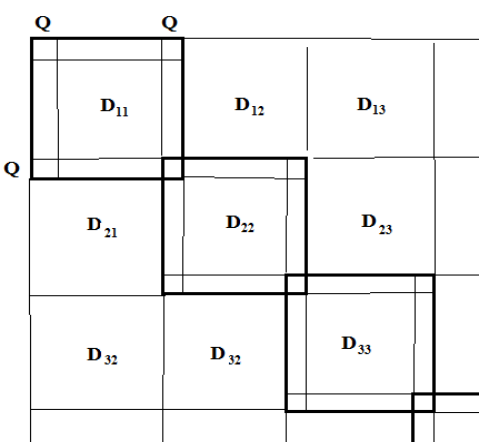

Рис. 5 - Структура системы разрешающих уравнений

Численная апробация. Предлагаемый универсальный подход решения задачи статического расчета шарнирно-сочлененных плит на упругом основании на вертикальную нагрузку численно реализуется для жестких плит (понтонов) на основании Винклера (пример 1), гибких железобетонных плит на упругом полупространстве (пример 2) и комбинированном основании Винклера - Когана.

Пример 1. Расчет системы из 7 прямоугольных жестких плит размерами 5 м 6,8м на основании Винклера (коэффициент постели $\left.10 \kappa H / M^{3}\right)$. Собственный вес плиты - 20 кН. Вторая, четвертая и шестая плита нагружены симметричной нагрузкой $R=600 \kappa H$. 
На рис. 6 показаны результаты расчета поперечных сил в соединительных шарнирах между плитами, реактивных давлений в зоне контактного взаимодействия, вертикальных (осадки) и угловых перемещений плит. Из условия симметрии результаты приведены для половины системы.
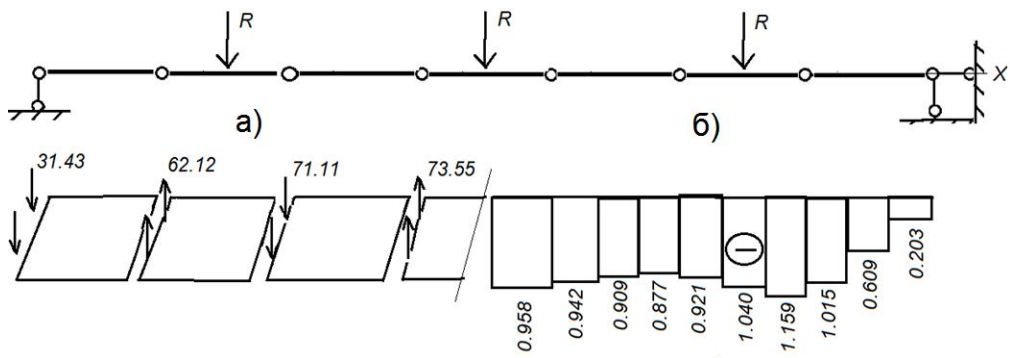

B)

г)

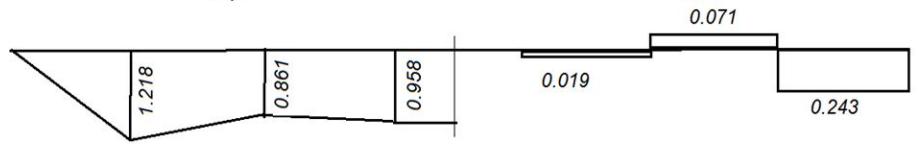

Рис. 6- Результаты расчета системы из 7 шарнирно-соединенных жестких плит (понтонов) на основании Винклера:

a) распределение поперечных сил в соединительных шарнирах $(\kappa \mathrm{H})$;

б) распределение реактивных давлений в контактной зоне $\left(\kappa H / \mathrm{M}^{2}\right)$;

в) вертикальные перемещения (осадки) плит (м);

г) угловые перемещения плит (рад) в точке $k$

Пример 2. В данном примере рассматривается расчет системы из 7 прямоугольных гибких плит размерами: длина $a=4 \mu$, ширина $b=3$ м на упругом полупространстве, со следующими характеристиками грунта $E_{0}=25$ МПа; $v_{0}=0.2 ; h_{0}=0.25$ м. Собственный вес плиты $-54 \mathrm{\kappa H}$.

Показатель гибкости определяется по формуле [2] и равен

$$
\beta=\frac{\pi E_{0} a^{3}}{\left(1-v_{0}^{2}\right) D}=44.5 .
$$

Вторая, четвертая и шестая плита нагружены симметричной нагрузкой $R=600 \kappa H$. Разбивка каждой плиты - на $3 \times 4$ равных прямоугольных участков. Общее число неизвестных - 121.

На рис. 7 и рис. 8 приведены результаты расчета для распределения поперечных сил в соединительных шарнирах, осадок центров плиты и усилий в связях Жемочкина. Из условия симметрии, как и в примеpe 1, результаты расчета приведены для половины системы. 

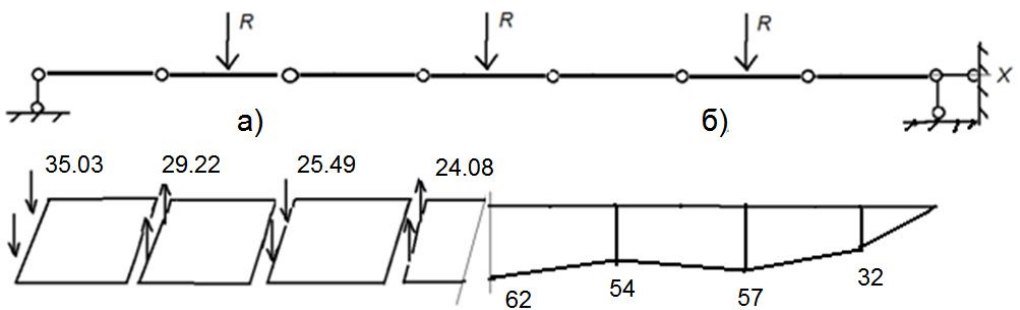

Рис. /- Результаты расчета системы из I шарнирно-соединенных гибких плит на упругом полупространстве:

а) распределение поперечных сил в соединительных шарнирах (кH);

б) осадки центров плиты (мм).

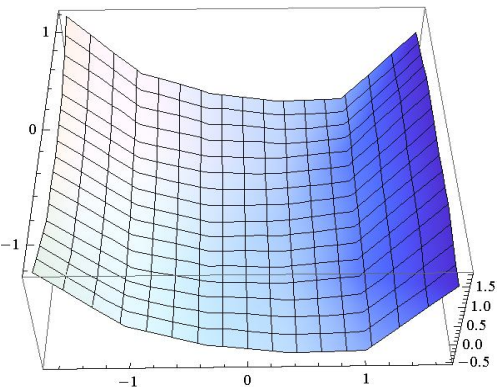

a)

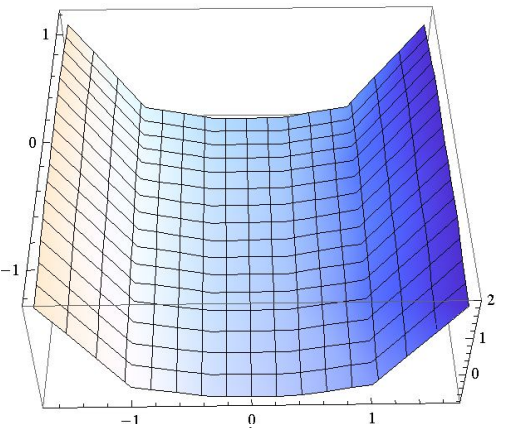

B)

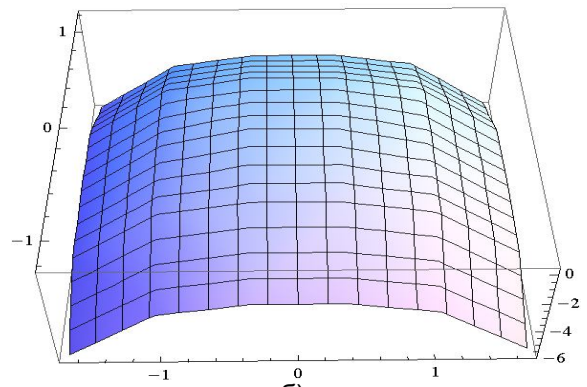

б)

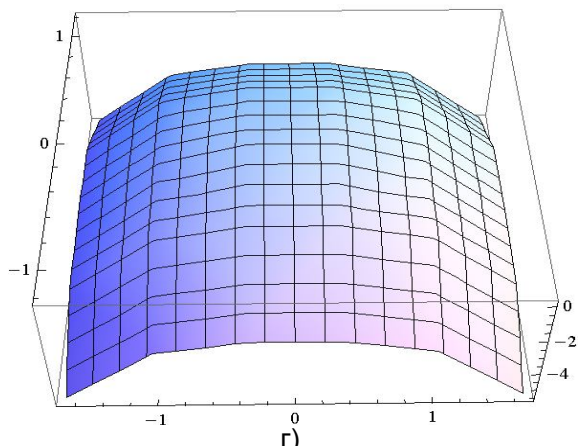

г)

Рис. 8 - Результаты расчета системы из 7 шарнирно-соединенных гибких плит: усилия в связях Жемочкина (10 кH)

а) в первой слева плите; б) во второй слева плите;

в) в третьей слева плите; г) в центральной плите

Пример 3. Расчеты выполнялись для системы из 7 шарнирно-соединенных дорожных железобетонных плит размером $4 M \times 3 м \times 0,18 m$ из тяжелого бетона $C^{20 / 25}$ на комбинированном основании Винклера- Когана со следующими характеристиками упругих слоев слоистого основания (рис. 3): 


$$
\begin{array}{lll}
E_{2}=25 M \Pi a ; & v_{2}=0.2 ; & h_{2}=0.25 \mathrm{M} ; \\
E_{1}=15 M \Pi a ; & v_{1}=0.25 ; & h_{1}=0.5 \mathrm{M} ; \\
E_{0}=12 M \Pi a ; & v_{0}=0.35 . &
\end{array}
$$

Внешняя нагрузка $Q=67,5 \kappa H$ распределена по площади $0,4 \mathrm{M} \times 0,4 \mathrm{M}$ и приложена в центре плиты. Собственный вес плиты - 20 кH.

Показатель гибкости прямоугольной плиты $\beta$ определяется по формуле [2], с учетом ее опирания через винклеровский слой комбинированного основания (щебень, с упругими параметрами $E_{2}, v_{2}$ ) на верхний слой двухслойного основания (песок, с упругими параметрами $E_{1}, v_{1}$ ), равен

$$
\beta=\frac{\pi E_{1} a^{3}}{\left(1-v_{1}^{2}\right) D}=218.745,
$$

где $a$ - ширина прямоугольной плиты в плоскости изгиба, $a=4 M ; D-$ цилиндрическая жесткость железобетонной плиты из тяжелого бетона класса $C^{20 / 25}\left(E_{b}=2,905 \cdot 10^{10} \Pi a\right), D=1470,66 \kappa H: м$.

По формуле (9) определяется коэффициент постели [4]. После подстановки получаем

$$
k=111,11 \text { МПа } / \text { м. }
$$

Вторая, четвертая и шестая плита нагружены симметричной нагрузкой $R=\mathrm{Q}=67,5 \kappa H$. Принята разбивка каждой плиты на $6 \mathrm{x8}$ равных прямоугольных участков. Общее число неизвестных - 121.

На рис. 9 и рис. 10 приведены результаты расчета системы из 7 шарнирно-соединенных дорожных железобетонных плит: распределение поперечных сил в соединительных шарнирах, осадки центров плиты, усилия в связях Жемочкина.

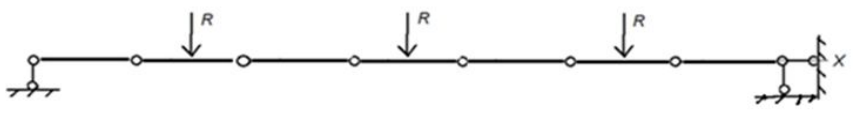

a)

б)

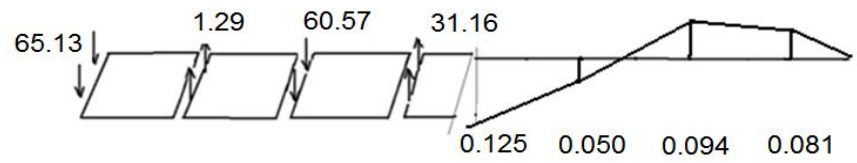

Рис. 9- Результаты расчета системы

из 7 шарнирно-соединенных дорожных плит:

а) - распределение поперечных сил в соединительных шарнирах (кН); б) - осадки центров плиты (мм) 


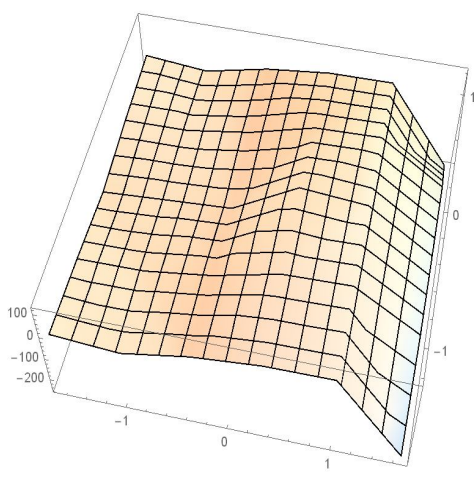

a)

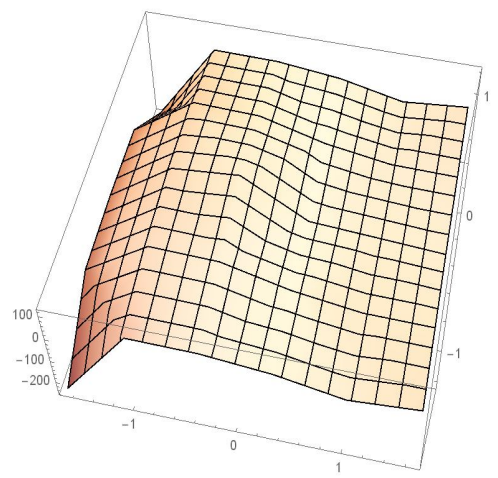

B)

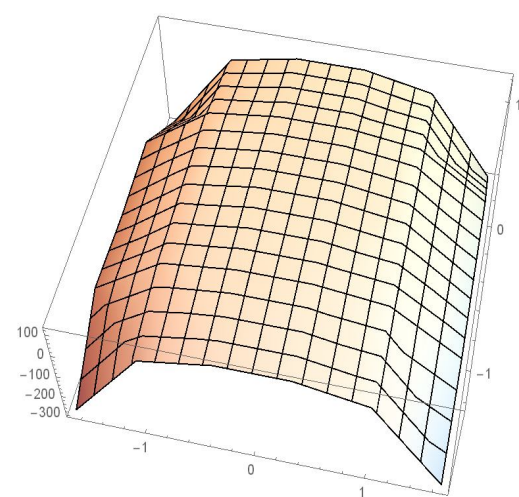

б)

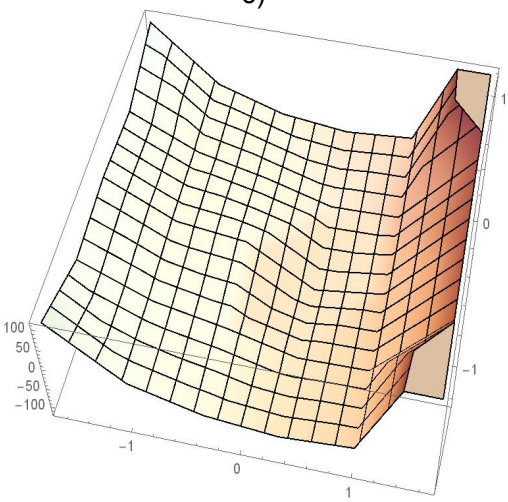

г)

Рис. 10 - Результаты расчета системы из 7 шарнирно-соединенных дорожных плит: усилия в связях Жемочкина (кН)

а) в первой слева плите; б) во второй слева плите;

в) в третьей слева плите; г) в центральной плите

Выводы. В работе разработана несложная универсальная методика решения контактных задач статического расчета системы шарнирносочлененных прямоугольных плит на упругом основании на вертикальную нагрузку. Методика основана на смешанном методе строительной механики и способе Б. Н. Жемочкина и может быть обобщена на любое число плит конечной жесткости и различные модели упругого основания. Отличие от стандартного способа Жемочкина заключается во введении дополнительных неизвестных поперечных сил в соединительных шарнирах и составлении условий равенства вертикальных перемещений в общих шарнирах смежных прямоугольных плит.

Полученные результаты могут быть непосредственно использованы при расчете сборных железобетонных дорожных и аэродромных плит (гибкие плиты), плит понтонных переправ (жесткие плиты). 


\title{
БИБЛИОГРАФИЧЕСКИЕ ССЫЛКИ
}

1. Босаков С. В., Зиневич С. И., Козунова О. В. Расчет шарнирно-соединенных прямоугольных жестких плит на основании Винклера // НТЖ Строительная механика и расчет сооружений. М. 2018. №3 (278). С. 8-10.

2. Босаков С. В., Зиневич С. И., Козунова О. В. Об одной модели упругого основания и ее использовании для расчета прямоугольной плиты на упругом основании // НТЖ Строительная механика и расчет сооружений. М. 2018. №4 (279). С. 2-5.

3. Босаков С. В.Статические расчеты плит на упругом основании. Мн.: БНТУ,2002. 128с.

4. Горбунов-Посадов М. И., Маликова Т. А., Соломин В. И.Расчет конструкций на упругом основании. М.: Стройиздат, 1984. 639 с.

5. Горбунов-Посадов М. И. Осадки фрундаментов на слое грунта, подстилаемом скальным основанием. М.: Гсостройиздат, 1946.

6. Жемочкин Б. Н., Синицын А. П. Практические методы расчета фундаментных балок и плит на упругом основании. М.: Стройиздат, 1962. 239с.

7. Коган Б. И. Напряжения и деформации многослойных покрытий // Тр. ХАДИ, 1953. Вып.14. С. 33-46.

8. Коренев Б. Г. Вопросы расчета балок и плит на упругом основании. М.: Стройиздат, 1954. 127 с.

9. Попов Г. Я. О расчете неограниченной шарнирно-разрезной балочной плиты, лежащей на упругом полупространстве // Изв. Вузов, Строительство и архитектуpa. 1959. №3. С. 25-33.

10. Ржаницин А. Р. Строительная механика. М.: Высшая школа, 1991. 439с.

11. Серебряный $\boldsymbol{P}$. В.Расчет тонких шарнирно-соединенных плит на упругом основании. М.: Стройиздат, 1962. 64c.

12. Юрьев А. Г. Балки и плиты с конструктивными и пластическими шарнирами на податливом основании. Харьков: Изд. Изв. Вузов. 1964.

УДК 624.13

${ }^{1}$ C. В. Босаков, д-р техн. наук. ${ }^{2}$ О. В. Козунова, канд. техн. наук

РОЗРАХУНОК ШАРНІРНО-СПОЛУЧНИХ ПРЯМОКУТНИХ ПЛИТ НА ПРУЖНІЙ ОСНОВІ

Розглянуто задачу статичного розрахунку шарнірно-сполучних в окремих точках прямокутних плит понтонних переправ на основі Вінклера, дорожніх та аеродромних плит на пружному півпросторі й комбінованій основі Вінклера Когана при довільному навантаженні. Сумісні плити з'єднані циліндричними шарнірами. Розрахунок виконано мішаним методом будівельної механіки 3 використанням способу Жемочкіна і врахуванням власної ваги кожної плити.

Ключові слова: статичний розрахунок, прямокутна плита, пружна основа, спосіб Жемочкіна.

UDC 624.13

\author{
${ }^{1}$ S. V. Bosakov Dr. Sci. (Tech.), ${ }^{2}$ O. V. Kozunova PhD (Tech.) \\ CALCULATION OF ARTICULATED RECTANGULAR PLATES \\ ON ELASTIC BASE
}


The paper deals with the problem of static calculation of articulated at individual points rectangular plates of pontoon crossings on the basis of Winkler, road and airfield plates on an elastic half-space and a combined Winkler-Kogan base on an arbitrary load. Adjacent plates are connected by cylindrical hinges at two points. The calculation is performed by a mixed method using the Zhemochkin method considering the weight of each plate.

Keywords: static calculation, rectangular plate, elastic base, Zhemochkin method.

The problems of calculation of articulated structures (beams, frames, plates) on elastic base have not been fully investigated to date. The authors are aware of the work of B. G. Koreneva [8], R. V. Silver [11], G. Ya. Popova [9], A. G. Yuriev [12], in which different methods were used to conduct theoretical studies on the calculation of articulated beams and plates on elastic base.

Static calculation of articulated plates on the elastic Winkler base was considered earlier by the authors in work [1]. In work [2] is given the model of the three-layer elastic base for calculation of the isolated road or airfield plate. The upper layer is the base of the Winkler and is located on a twolayer base of the Kogan. The calculation of an isolated rectangular plate on this model with elastic base is made with Zhemochkin method to the load applied from the central wheel of the machine. This took into account the own weight of the plate.

The following is a general approach for the calculating of articulated rectangular plates on a three-layer basis, based on the mixed method of structural mechanics [10] and the Zhemochkin method[6], which allows to calculate articulated plates or beams of any shape and rigidity on different elastic base models on an arbitrary vertical load. The numerical implementation of the proposed approach is made on seven articulated rectangular flexible plates on a three-layer base.

It should be noted, that earlier in work [8], the question of the possibility of using the Zhemochkin method in solving such problems was discussed.

\section{REFERENCES}

1. Bosakov S. V., Zinevich S. I., Kozunova O. V. Pivotally connected rigid Calculation of rectangular plates based on Winkler // Scientific-technical journal: Structural mechanics and calculation of structures. Moscow. 2018. No 3 (278). P.8-10. (in Russian).

2. Bosakov S. V., Zinevich S. I. Kosunova O. V. A model for the elastic Foundation and its use for the calculation of rectangular plates on elastic foundations / / Scientifictechnical journal: Structural mechanics and calculation of structures. Moscow. 2018. No 4 (279). P.2-5. (in Russian).

3. Bosakov S. V. Static calculations of plates on an elastic base. Meganewton, BNTU, 2002. 128c. (in Russian).

4. Gorbunov-Posadov M. I., Malikova T. A., Solomin, V. I. Analysis of structures on elastic Foundation. Moscow: Stroyizdat, 1984. 639 p. (in Russian).

5. Gorbunov-Posadov M. I. Sediments of foundations on a layer of soil, underlain by a rocky base. Moscow: Gosstroyizdat, 1946. (in Russian).

6. Zhemochkin B. N., Sinitsyn. A. P. Practical methods of calculation of Foundation beams and plates on an elastic base. Moscow: Stroyizdat, 1962. 239c. (in Russian). 
7. Kogan B. I. Stresses and deformations of multilayer coatings // Tr. HADI, 1953. Vol.14. P. 33-46. (in Russian).

8. Korenev B. G. Questions of calculation of beams and plates on the elastic basis. M., Stroyizdat, 1954. 127 p. (in Russian).

9. Popov G.Ya. On the unbounded calculation of a hinged-split beam plate lying on an elastic half-space // WPI. Universities, Construction and architecture. 1959. No 3, P. 2533. (in Russian).

10. Rzhanitsin A. R. Construction mechanics. Moscow: High school. 1991. 439 p. (in Russian).

11. Serebryany R. B. Calculation of thin hinged plates on an elastic base. M., Stroyizdat, 1962.64 p. (in Russian).

12. Yuriev A. G. Beams and plates with constructive and plastic Charny-Rami on compliant substrate. Kharkov: Ed. WPI. Higher educational. 1964. (in Russian).

Друкується за рекомендацією програмного комітетуVII Міжнародної науковотехнічної конфреренції "Актуальні проблеми прикладної механіки та міцності конструкцій», 23-26 травня 2019 р. м. Запоріжжя.

${ }^{1}$ Белорусский национальный

Техничкий університет, РУП «Институт БелНИИС», Минск, Республика Беларусь

${ }^{2}$ УО «Белорусский государственный университет транспорта», Гомель, Республика Беларусь 